\title{
Untargeted metabolomics confirms and extends the understanding of the impact of aminoimidazole carboxamide ribotide (AICAR) in the metabolic network of Salmonella enterica
}

\author{
Jannell V. Bazurto ${ }^{1}$, Stephen P. Dearth ${ }^{2}$, Eric D. Tague ${ }^{2}$, Shawn R. Campagna ${ }^{2}$ and Diana M. Downs ${ }^{1, *}$ \\ ${ }^{1}$ Department of Microbiology, University of Georgia, Athens, GA 30602. \\ ${ }^{2}$ Department of Chemistry, University of Tennessee, Knoxville, TN 37996. \\ * Corresponding Author: \\ Diana M. Downs, Department of Microbiology, University of Georgia, 120 Cedar St., Athens, GA 30602; PH: 706-542-1434; FAX: 706- \\ 542-2674; E-mail: dmdowns@uga.edu
}

\begin{abstract}
In Salmonella enterica, aminoimidazole carboxamide ribotide (AICAR) is a purine biosynthetic intermediate and a substrate of the AICAR transformylase/IMP cyclohydrolase (PurH) enzyme. When purH is eliminated in an otherwise wild-type strain, AICAR accumulates and indirectly inhibits synthesis of the essential coenzyme thiamine pyrophosphate (TPP). In this study, untargeted metabolomics approaches were used to i) corroborate previously defined metabolite changes, ii) define the global consequences of AICAR accumulation and iii) investigate the metabolic effects of mutations that restore thiamine prototrophy to a purH mutant. The data showed that AICAR accumulation led to an increase in the global regulator cyclic AMP (CAMP) and that disrupting central carbon metabolism could decrease AICAR and/or cAMP to restore thiamine synthesis. A mutant (icc) blocked in cAMP degradation that accumulated cAMP but had wild-type levels of AICAR was used to identify changes in the purH metabolome that were a direct result of elevated CAMP. Data herein describe the use of metabolomics to identify the metabolic state of mutant strains and probe the underlying mechanisms used by AICAR to inhibit thiamine synthesis. The results obtained provide a cautionary tale of using metabolite concentrations as the only data to define the physiological state of a bacterial cell.
\end{abstract}

doi: $10.15698 /$ mic2018.02.613 Received originally: 08.09.2017; in revised form: 03.11.2017, Accepted 07.11.2017, Published 22.11.2017.

Keywords: aminoimidazole carboxamide ribotide (AICAR), cyclic AMP (cAMP), adenylate cyclase (CyaA), cyclic AMP phosphodiesterase (Icc), purinehistidine-thiamine (PHT) metabolic network, thiamine biosynthesis, untargeted metabolomics.
Abbreviations:
AIRCAR-aminoimidazole carboxamide ribotide, AIRCARs - AIRCAR riboside, CoA-coenzyme $A$, $P H T$ - purine-histidine-thiamine, TPP-thiamine pyrophosphate, VIP - variable importance in projections.

\section{INTRODUCTION}

In Salmonella enterica, investigating metabolic redundancy in the thiamine biosynthetic pathway is a well-established model system for identifying complex metabolic interactions $[1,2]$. Studies with this system have characterized numerous connections between thiamine synthesis and diverse areas of metabolism including, amino acid biosynthesis [3-6], metabolite detoxification systems $[5,7,8]$ and cofactor biosynthesis [9-11]. By characterizing individual points of metabolic integration, these studies have ex- panded our understanding of the purine-histidine-thiamine (PHT) metabolic network and how it interacts with the metabolic network at large (Figure 1). Research with this model system has defined non-canonical roles of specific metabolites, elucidated catalytic mechanisms of biosynthetic enzymes and demonstrated the physiological relevance of redundant pathways in related organisms [12].

Aminoimidazole carboxamide ribotide (AICAR) is a prominent component of the PHT metabolic network and has an increasingly recognized, and complex, role within it 


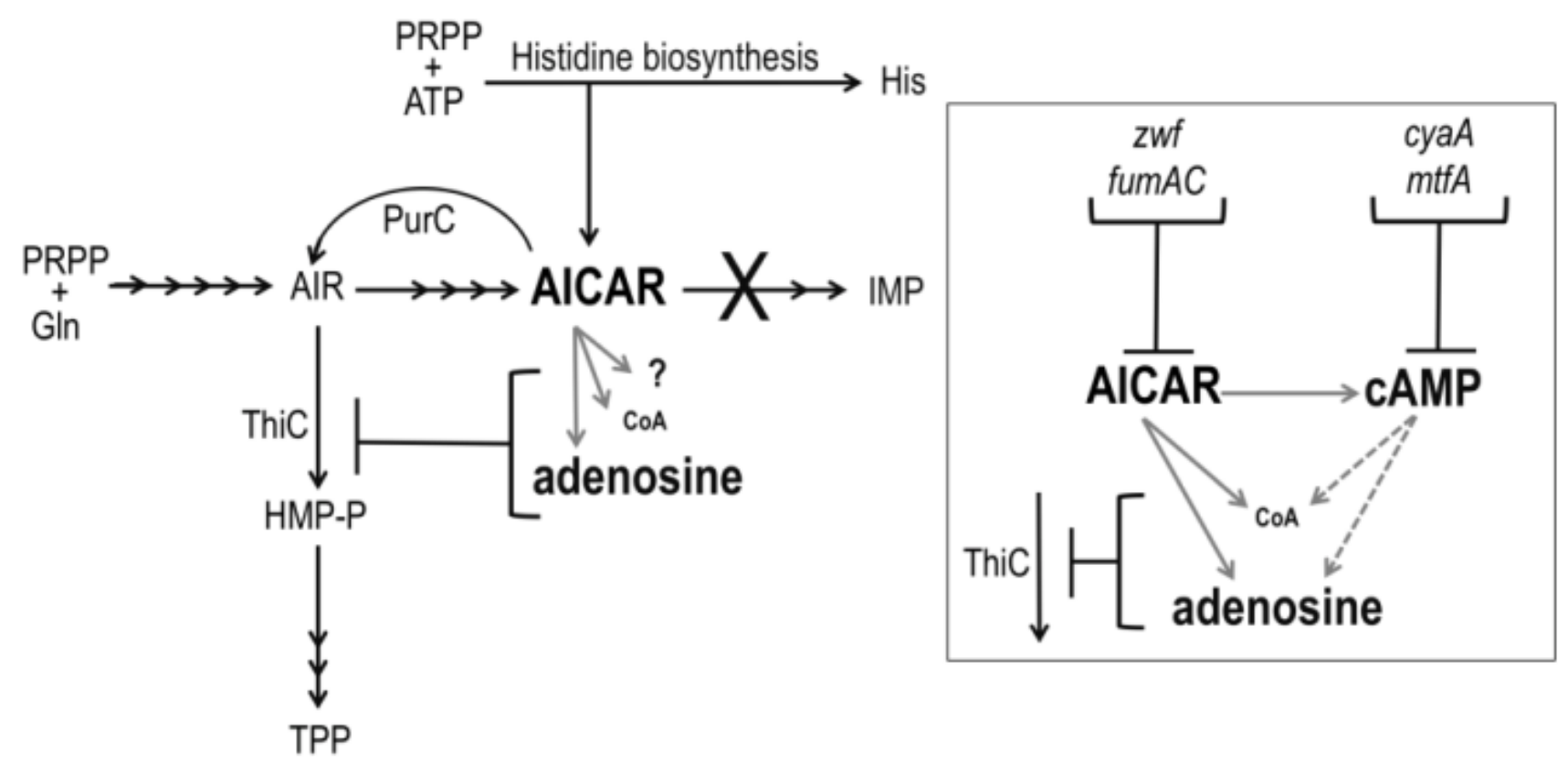

FIGURE 1: The role of AICAR in the purine histidine thiamine (PHT) metabolic network of S. enterica. Solid black arrows represent biochemical reactions; corresponding gene products are beside the reaction they catalyze. AICAR-mediated points of metabolic crosstalk that impact thiamine synthesis (ThiC activity) are indicated with gray arrows. Inset: AICAR accumulation leads to elevated cAMP. Elevated AICAR and CAMP independently decrease pantothenate $(\mathrm{COA})$ pools and increase adenosine pools demonstrating that these pools are subject to direct (solid lines) and indirect (dashed lines) effects of AICAR accumulation. These altered pools potentially contribute to decreased ThiC activity; loss of function mutations ( $z w f$, fumAC, cyaA, $m t f A$ ) modulated AICAR and/or cAMP levels and restored thiamine synthesis.

(Figure 1). Though known primarily for being a purine biosynthetic intermediate, AICAR is also a histidine biosynthetic byproduct, a precursor to thiamine in some genetic contexts and an indirect inhibitor of thiamine synthesis. Thiamine pyrophosphate (TPP) is a nearly ubiquitous coenzyme that until recently was believed to be essential for central metabolic processes of all organisms [13]. TPP is composed of separately synthesized thiazole and pyrimidine moieties. In bacteria and plants the pyrimidine moiety (4-amino-5-hydroxymethyl-2-methylpyrimidine, HMP) is synthesized in a pathway that branches off of the purine biosynthetic pathway. Although AICAR is synthesized beyond the last common intermediate of purine-HMP synthesis, aminoimidazole ribotide (AIR), it has long been known to abolish HMP synthesis when it accumulates intracellularly [14, 15], and previous work has demonstrated that it indirectly inhibits the ThiC enzymatic step [16] by more than one mechanism [10] (Figure 1).

In S. enterica, mutants that accumulate AICAR have been generated by eliminating AICAR transformylase/IMP cyclohydrolase (PurH, EC:2.1.2.3/3.5.4.10), a bifunctional enzyme that catalyzes the last two steps in purine biosynthesis and the only known consumer of AICAR. Suppressor strains of the purH mutant that regained the ability to synthesize thiamine (HMP) were used to determine that AICAR (and its riboside derivative AICARs) is a direct inhibitor of pantoate- $\beta$-alanine ligase (PanC, EC:6.3.2.1). This inhibition decreased coenzyme A (COA) levels to $33 \%$ of wild-type [10]. CoA has an elusive role in HMP synthesis but data has shown that HMP synthase (ThiC, EC:4.1.99.17) is sensitive to decreased CoA levels in vivo $[9,16]$ and suggest $\mathrm{CoA}$ contributes to the reducing environment of the cell [17] that may protect ThiC's Fe-S cluster from oxidation $[11,18]$. Further nutritional work suggested that AICAR had at least one additional negative impact on ThiC activity [10] and demonstrated that lesions in multiple genes involved in central carbon metabolism (cyaA, gdhA, mtfA, zwf and fumAC) could restore thiamine synthesis in a purH mutant [19]. These data indicated that more than one metabolic pathway important for ThiC function was disrupted by AICAR accumulation and suggested that lesions in $c y a A$, $g d h A, m t f A, z w f$ and fumAC modulated AICAR levels and/or neutralized its impact on ThiC activity.

Genetic and biochemical data from previous studies have defined numerous roles for AICAR in metabolism [10, 20-28]. The integration of the metabolic network suggested that AICAR accumulation would have a number of targets, each with their own downstream effects. Herein we investigate the global impact of elevated AICAR levels in $S$. enterica with untargeted metabolomics approaches. This study obtained metabolomics data that highlight the broad reach of AICAR in unexpected and distinct areas of metabolism. Importantly, this work demonstrates the potential, and describes caveats, of combining genetic analyses and metabolomics to deconstruct pleiotropic network perturbations in bacteria. 
TABLE 1. Important metabolites as determined by PLSDA.

\begin{tabular}{|c|c|c|c|c|c|}
\hline \multicolumn{2}{|c|}{ Exponential } & \multicolumn{2}{|c|}{ Early Stationary } & \multicolumn{2}{|c|}{ Late Stationary } \\
\hline Metabolite & VIP & Metabolite & VIP & Metabolite & VIP \\
\hline AICAR & 7.3736 & Acetyl-P & 1.5362 & Acetyl-P & 1.4554 \\
\hline AICARs & 3.1681 & AICAR & 6.5634 & Aconitate & 1.3504 \\
\hline ATP & 1.2336 & AICARs & 1.4952 & AICAR & 3.0974 \\
\hline Betaine/Val & 3.5394 & Asp & 1.5125 & Asp & 1.7768 \\
\hline Deoxyribose-P & 1.2292 & Betaine/Val & 5.5507 & Betaine/Val & 7.2269 \\
\hline 2,3-Dihydroxybenzoate & 1.6171 & Citrate & 2.5485 & Citrate & 3.8841 \\
\hline Homoserine/Thr & 1.0629 & 2,3-Dihydroxybenzoate & 2.2062 & Cyclic AMP & 1.0003 \\
\hline $\begin{array}{l}\text { 2-Hydroxy-2- } \\
\text { methylbutanedioic acid }\end{array}$ & 1.3098 & Glu $^{1}$ & 2.1229 & Deoxyinosine & 1.3386 \\
\hline Isopropylmalate & 3.9679 & $\mathrm{Glu}^{2}$ & 1.5235 & Dimethylglycine & 3.1393 \\
\hline Ketoisovalerate & 1.2343 & Hydroxyisocaproate & 1.4819 & Glu $^{1}$ & 3.4531 \\
\hline Malate $^{1}$ & 1.1813 & Isopropylmalate & 3.3397 & $\mathrm{Gln}$ & 1.0348 \\
\hline Malate $^{2}$ & 1.1813 & Leu/Ile & 1.5560 & Glycerate & 1.4783 \\
\hline Methylmalonate & 3.1222 & Malate $^{1}$ & 3.6822 & Hydroxyisocaproate & 1.8499 \\
\hline N-acetylglutamate & 4.9175 & Malate $^{2}$ & 3.0078 & $\begin{array}{l}\text { 2-Hydroxy-2- } \\
\text { methylbutanedioic acid }\end{array}$ & 1.9445 \\
\hline $\mathrm{NADH}$ & 1.1018 & Methylmalonate & 2.2483 & Hypoxanthine & 1.5394 \\
\hline N-carbamoyl-L-Asp & 1.1942 & N-acetylornithine & 2.0604 & Isopropylmalate & 2.6574 \\
\hline Orotate & 1.4249 & N-carbamoyl-L-Asp & 1.3100 & Ketoisovalerate & 1.3586 \\
\hline 6-Phosphogluconate & 1.5895 & Orotate & 1.3731 & Lipoate & 1.7865 \\
\hline 3-Phosphoglycerate & 1.2241 & Pantothenate & 2.0429 & Malate $^{1}$ & 2.9740 \\
\hline Pyroglutamate & 1.5009 & Pyroglutamic acid & 1.1308 & Malate $^{2}$ & 2.6831 \\
\hline Ser & 1.2722 & Pyrophosphate & 1.2704 & Pantothenate & 2.8073 \\
\hline UDP-D-glucose & 1.5608 & UDP-glucose & 1.4248 & Phe & 1.1058 \\
\hline $\begin{array}{l}\text { UDP-N-acetyl- } \\
\text { glucosamine }\end{array}$ & 1.0165 & & & Pyroglutamic acid & 1.0548 \\
\hline
\end{tabular}

Variable importance in projections (VIP) scores for the three growth phases were tested. Metabolites that have VIP score greater than 1 are indicated. Metabolites with VIPs $>1$ in 2 growth phases are bolded; those with VIPs $>1$ in 3 growth phases are bolded and boxed. The metabolites denoted as Glu ${ }^{2}$ and Malate ${ }^{2}$ are isomers of the listed compound and have an undetermined structure.

\section{RESULTS AND DISCUSSION}

Rationale for using global approaches to dissect the metabolic impact of AICAR

Extensive in vivo genetic and biochemical analyses have probed the structure of the metabolic network consisting of purine, histidine and thiamine biosynthetic pathways (PHT node) [3, 10, 16, 29-34]. While these approaches have provided significant metabolic and mechanistic insights, studies of the PHT system have reached the point where continued genetic analyses are laborious and unlikely to efficiently answer questions about metabolic network structure and integration. The PHT node was used as a test case for the implementation of metabolomics approaches to generate testable hypotheses to extend our understanding of the subtleties associated with metabolic integration.

An in-frame, non-polar deletion of purH in S. enterica lacks AICAR transformylase and IMP cyclohydrolase activities. In S. enterica, a purH mutant is auxotrophic for pu- rines and thiamine, due to elimination of the last two purine biosynthetic reactions, and inhibitory effects of AICAR accumulation on ThiC, respectively $[10,14,15]$. A number of nutritional supplements and suppressor mutations that restore thiamine-independent growth of a purH mutant have been described $[10,19]$. Of specific interest was the additive effect of methionine and pantothenate, that demonstrated that the AICAR-mediated inhibition of pantothenate synthesis was not the only perturbation that constrained thiamine synthesis when AICAR accumulated [10]. Null mutations in genes involved in pathways of central carbon metabolism alleviated constraints on thiamine synthesis [19] and added to the growing body of evidence that suggested that AICAR accumulation perturbed cellular homeostasis in a number of areas that then, directly or indirectly, compromised thiamine synthesis. The impact that AICAR accumulation has on cellular processes (in a range of organisms) such as purine synthesis, phosphate 

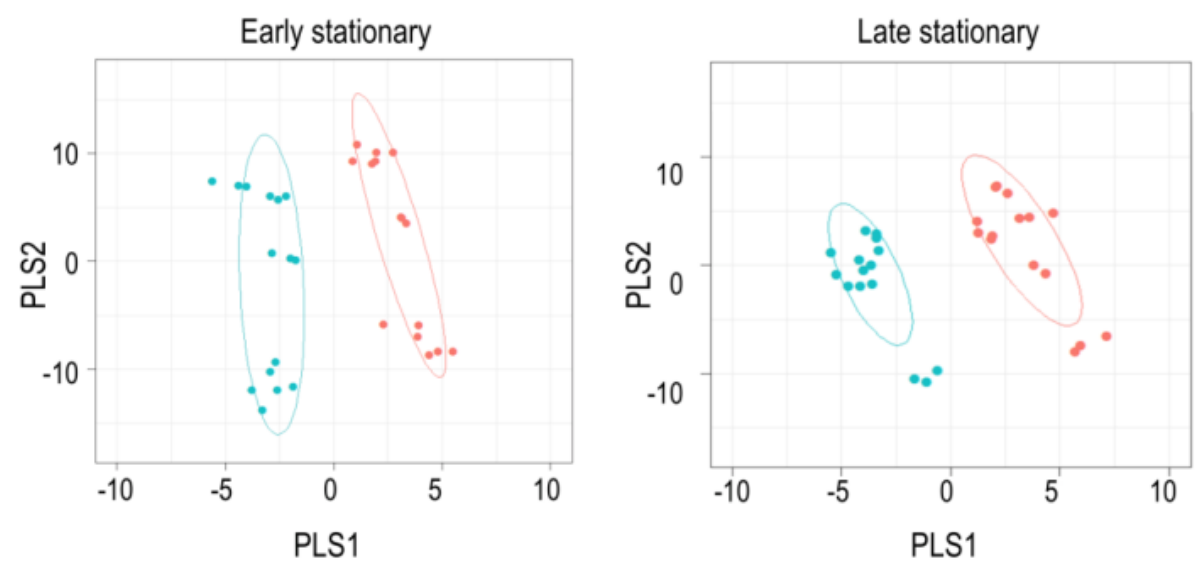

FIGURE 2: PLSDA of WT and purH mutant metabolomes. Untargeted metabolomics was performed on wild-type and purH mutant of $S$. enterica grown in glucose minimal medium supplemented with adenine and thiamine. Partial Least Square Discriminate Analysis (PLSDA) was used as a multivariate analysis tool to provide insight into the unique metabolic fingerprint of the purH mutant. Each dot represents one experimental replicate; WT is in cyan, the purH mutant is in peach. Confidence intervals are shown by the colored ellipses. utilization, energy homeostasis (including gluconeogenesis and fatty acid synthesis) and one carbon metabolism has been highlighted with various studies [10, 20-28].

\section{A purH mutation results in global metabolic changes}

An untargeted metabolomics approach was used in a proof-of-principle experiment to analyze a strain lacking PurH. To validate the use of this approach in probing metabolism, it was critical that the metabolic features that have been described for purH mutants were captured in this experiment. The metabolome of a purH mutant was compared to that of the isogenic parental strain (wild-type) in different growth stages by taking samples at mid-log $\left(\mathrm{Abs}_{650} \sim 0.475\right)$, early stationary $\left(\mathrm{Abs}_{650} \sim 1.5\right)$ and late stationary $\left(\mathrm{Abs}_{650} \sim 1.4\right)$ phase. Reproducibility of stationary phase results was rigorously queried by repeating the same experiment on two sequential days and then months later on three sequential days. In each experiment, biological triplicates were used. Water-soluble metabolites were extracted from wild-type (DM10000) and purH mutant (DM12239) strains of S. enterica and analyzed via UPLC-MS, using slight modifications of previously established methods $[35,36]$. The metabolomics data obtained from 15 samples ( 3 biological replicates $\times 5$ experimental replicates) across stationary growth phases and 3 samples ( 3 biological replicates $\times 1$ experiment) during exponential growth were used to identify metabolic changes that resulted when the PurH-catalyzed step in purine biosynthesis was blocked.

Using Metabolomic Analysis and Visualization Engine (MAVEN) software developed by Clasquin et al., approximately 140-180 metabolites were identified in each experiment [37]. Both multi- and single-variate approaches were used to analyze the data. Multivariate analysis using Partial Least Square Discriminate Analysis (PLSDA) resulted in the visual separation of the metabolomes in multidimensional space, demonstrating clearly that the metabolite profiles of a purH mutant are considerably different when compared to those of wild-type (Figure 2, Table 1 ). In early stationary phase, 22 metabolites were determined to be significant drivers of class separation, and 23 were significant in both late stationary and exponential phase. Five of these me- tabolites were common among the three growth phases, supporting the conclusion that these differences are linked to genotype not environmental factors. In particular, the class separation was conserved despite replicate experiments being done months apart. Table 1 shows all the variable importance in projections (VIP) scores $>1$ from each PLSDA. Over half of the metabolites with a VIP of $>1$ are present in at least two of the growth stages. Importantly, AICAR was the predominant driver of separation ( $\mathrm{VIP}=3-7)$. This result was gratifying in that it reflected the major change expected in a purH mutant that lacks the AICAR transformylase enzyme. The data further showed that metabolites not directly related to the $\mathrm{purH}$ mutation contributed to the separation of the metabolomes.

Despite the clear separation of the metabolite profiles between strains (Figure 2, Table 1), there were variations in the detection of some metabolites between experiments. Most notably, the second set of experiments ( 3 sequential days) consistently detected $\sim 20 \%$ more metabolites than the first set of experiments ( 2 sequential days). The few differences in detection between growth phases could reasonably reflect physiological fluctuations that decreased metabolite concentrations below the limit of detection, and thus these were not concerning. In all cases, the parameter of interest was the ratio of metabolite concentrations (pool sizes) present in the wild-type vs. purH mutant strains; no attempts were made to assess the absolute concentrations of metabolites. The distribution of calculated fold changes (purH/WT) and the relative abundance of statistically significant differences (as determined by $p$ value from an unpaired Student's t-test) observed in a given experiment are shown in Figure 3. Here, each data point is the ratio for one metabolite and the horizontal line represents the threshold above which $p$-values indicated a significant difference $(<0.05)$. These data suggested that a large number of metabolites are altered in a purH mutant; this result was surprising since bacteria are proficient at restoring cellular homeostasis after a perturbation [38-40]. In each experiment, across all growth phases, $25-30 \%$ of the detected metabolites were differentially present in wild-type and purH mutant strains. In fact, this may be an under estimate since this number represents only metabo- 


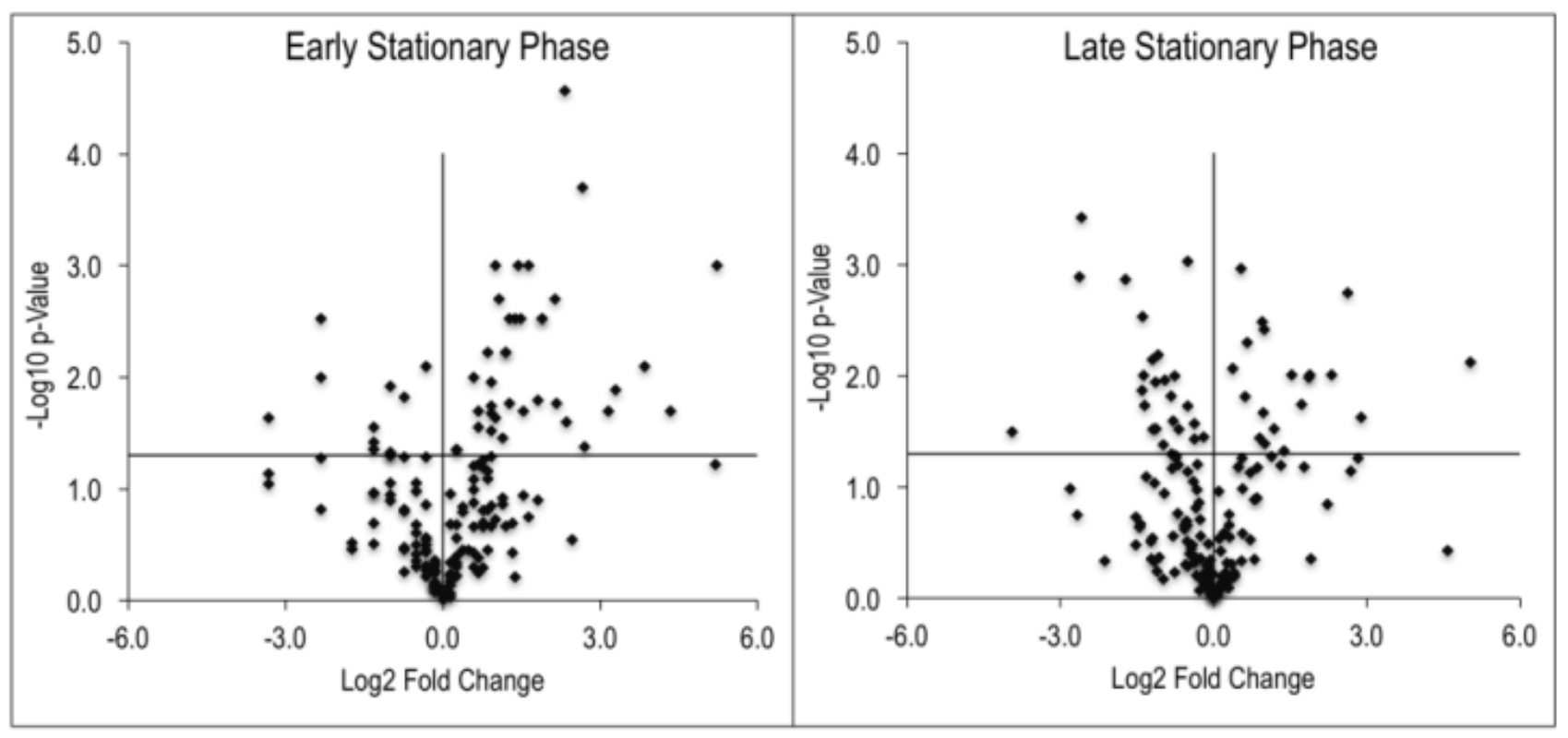

FIGURE 3: The metabolome of a purH mutant is significantly altered. Untargeted metabolomics was performed on wild-type and purH mutant of $S$. enterica grown in glucose minimal medium supplemented with adenine and thiamine. Relative concentrations of metabolites were determined by calculating the ratio of the average MS ion intensities (peak areas) in wild-type and purH strains (purH/WT). Here the log transformed fold changes $(\log 2)$ and $p$-values $(-\log 10)$ were plotted against each other, distributing the metabolite pool decreases and increases to the left and right of the $x=0$ point, respectively. The horizontal line $(y=1.3)$ indicates the threshold above which $p$-values were considered statistically significant $(<0.05)$. These data are derived from one representative experiment (Table S2).

lites where statistical significance of a non-one ratio could be clearly assigned. To distill true changes in the metabolome, statistically significant changes identified in early and late stationary phases were compared across 3 experiments (three sequential days). When fold-change trends were maintained across experiments, these changes were considered experimentally validated (Table S3). These data demonstrated that several metabolite pools involved in carbon, cofactor, amino acid, purine and pyrimidine metabolism were altered in a purH mutant and indicated the range of cellular processes that were perturbed by AICAR accumulation (Figure 4, Table S3). The variation between the relative levels of metabolites between experiments suggested that untargeted metabolomics was not appropriate as a stand-alone approach to quantify all metabolic differences between strains at the level of replication presented herein, but valuable information on key drivers of metabolic differentiation between the strains were detected. Fewer reports exist of metabolomics investigations in bacteria than in other organisms, and this observation coupled with these data may highlight difficulties in performing metabolomics in these microorganisms due to the coupled biological and technical variability when measuring bacterial metabolites.

\section{Untargeted metabolomics captures known metabolic features of a purH mutant}

Beyond the overall differences between the $\mathrm{purH}$ and wildtype metabolomes depicted in Figures 2-4, the datasets were culled for evidence that they reflected specific prior biological knowledge. In this vein, key metabolites expected to be different in a purH mutant (AICAR, AICARs, pantothenate, adenosine) were evaluated in the untargeted metabolomics datasets (Table 2) [10, 14, 15, 20]. In every sample, across experiments and growth phases, AICAR pools were considerably higher in the $\mathrm{purH}$ mutant and the data were statistically significant in 5 of 5 early stationary phase and 2 of 5 late stationary phase samples. Gratifyingly, the ratio of AICAR in a purH mutant relative to wild-type during mid-log phase (65.0-fold, p-value 0.007 ) was on par with a previous report where investigators showed a 10fold increase in AICAR levels within just an hour of antifolate (PurH inhibition) treatment [41]. The AICAR riboside (AICARs) is the predominant form of AICAR that accumulates in a purH mutant [42]. In two experiments AICARs was not detected in wild-type or purH samples. In the remaining 3 experiments, however, the expected accumulation of AICARs was observed. Although all experiments showed a $>90$-fold increase of AICARs in purH vs. wild-type in early stationary phase, statistical analysis attributed significance to the values in just 1 of 3 the experimental replicates. This result indicated that not all expected metabolic changes would be identified by our methods of data analyses, even in the face of gross metabolite changes. A point of concern was the inability to verify the statistical significance of the data with multiple experimental repetitions.

Data in Table 2 showed there was a dramatic decrease ( 10 -fold) in AICAR and AICARs accumulation in the purH mutant in late stationary phase. Analyses of the culture supernatants in late stationary phase showed an increase 


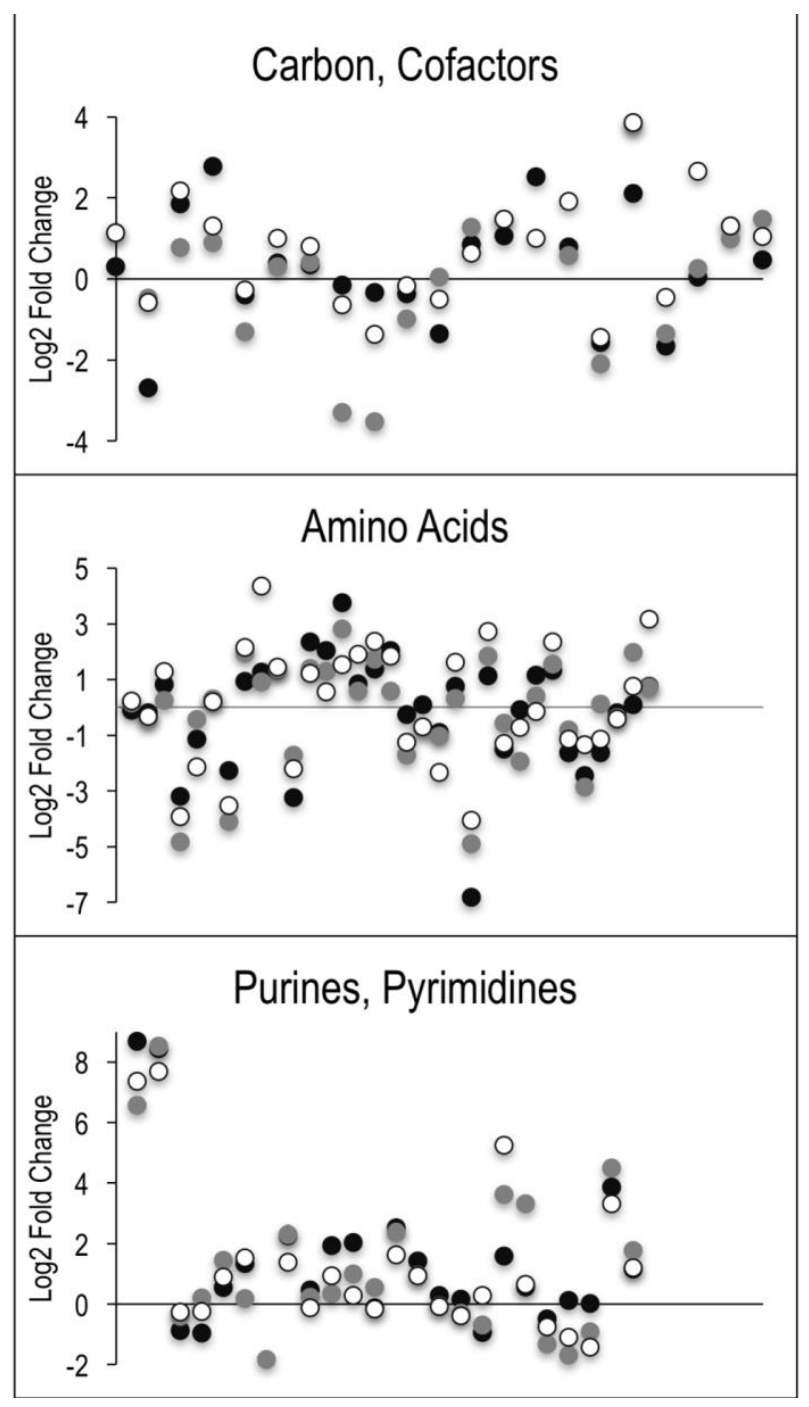

FIGURE 4: AICAR perturbs various areas of metabolism. Untargeted metabolomics was performed on wild-type and $\mathrm{purH}$ mutant of $S$. enterica grown in glucose minimal medium supplemented with adenine and thiamine. Relative concentrations of metabolites were determined by calculating the ratio of the average MS ion intensities (peak areas) in wild-type and purH strains (purH/WT). Here the log transformed fold changes (Log2) of metabolites with statistically significant differences were plotted to distribute the decreases and increases (below and above) the $y$ axis. Each point along the $\mathrm{x}$-axis represents a metabolite. Each of 3 experimental replicates are differentiated by color. Specific metabolites, fold changes and $p$-values are provided in Table S3.

in AICAR in the purH vs wild-type supernatants (53.9-fold, p-value 0.002). Together these data suggested AICAR and AICARs were secreted in late stationary phase, consistent with a previous report of the accumulation of AICAR/AICARs in culture medium of the purH mutants [15, 42].

Genetic analyses led to the finding that during exponential growth total CoA levels in a purH mutant were decreased to $33 \%$ of wild-type, due to inhibition of a pantothenate biosynthetic enzyme by AICAR [10]. Although CoA was not detected by the metabolomics platform used here, pantothenate was reliably detected and consistently present at lower levels in a purH mutant (Table 2). Although pantothenate was only detected in 3 of 5 experiments, in these replicate experiments the pantothenate levels averaged $30 \%$ of wild-type during early stationary phase ( 3 of 3 , p-value $<0.05$ ) and $40 \%$ during late stationary phase ( 2 of 3 , $p$-value $<0.05)$ and were thus well-correlated with altered CoA levels.

Finally, during growth on adenine (as cells are grown here) adenosine pools are expected to be inflated in a purH mutant as a result of direct inhibition of adenosine deaminase by AICAR [20]. Increased adenosine pools in a purH mutant were captured by the metabolomics platform in all data from early stationary phase (avg. 1.7-fold increase) with modest statistical significance (Table 2). Recently, adenosine was identified as a direct inhibitor of ThiC [43], thus these elevated pools may further constrain thiamine synthesis in a purH mutant. These data supported the ability of untargeted metabolomics to detect relevant metabolite changes, and again emphasized the problematic issue of statistical significance that was often lacking despite clear non-one ratios between the strains.

On several occasions our analyses failed to assign statistical significance to changes that were known to occur. In some instances, such as AICAR accumulation in a purH mutant in late stationary phase, slightly more lenient $p$ values would have resolved this (Table 2). In other cases, such as AICARs and adenosine (Table 2), p-values were exceedingly high and replicate experiments were critical in identifying a trend with confidence. Herein we chose to be conservative and prioritized eliminating false positives with a stringent $p$-value cut off. However, implementing a fold change cutoff (e.g., >2.0), in addition to the t-test, might more completely capture relevant changes [44].

A false positive discovery rate of statistically significant changes was evaluated by comparing the purH mutant used throughout this study to an isogenic strain that contained a neutral transposon insertion in two sequential experiments ( 3 biological replicates per experiment). The number of statistical significant differences between these presumably identical strains were 3.6 and $12.1 \%$ of those found in the same experiments when the purH mutant was compared to wild-type (data not shown). When these differences were further evaluated to see if their respective trend held up in the second experiment, half of them did not.

In total, the data in Table 2 included metabolites that had both small (pantothenate, adenosine) and large (AICAR, AICARs) fold changes in a purH mutant compared to wildtype. Significantly, these metabolites were biologically relevant, and each had been shown to differ between a purH mutant and wild-type in experiments that used traditional reductionist approaches. Thus, while some caveats with the untargeted metabolomics approach were raised, the data were consistent with previous knowledge and provided confidence in the metabolite trends detected with an untargeted metabolomics approach. 
TABLE 2. Metabolomics captures known metabolic changes of a purH mutant.

\begin{tabular}{|c|c|c|c|c|c|}
\hline & & \multicolumn{2}{|c|}{ Early stationary } & \multicolumn{2}{|c|}{ Late stationary } \\
\hline & Exp. $^{a}$ & Fold Change $^{b}$ & p-value ${ }^{c}$ & Fold Change & $p$-value \\
\hline \multirow[t]{5}{*}{ AICAR } & 1 & 346.6 & $0.009 * * *$ & 16.8 & $0.116-$ \\
\hline & 2 & 367.2 & $0.003^{* * *}$ & 34.1 & $0.032 * *$ \\
\hline & 3 & 206.0 & $0.017 * *$ & 32.7 & $0.007 * * *$ \\
\hline & 4 & 124.9 & $0.020 * *$ & 82.0 & $0.053-$ \\
\hline & 5 & 133.0 & $0.033^{* *}$ & 47.9 & $0.055-$ \\
\hline \multirow[t]{5}{*}{ AICARs } & 1 & 407.2 & $0.194-$ & 2.8 & $0.011 * *$ \\
\hline & 2 & 94.4 & $0.027^{* *}$ & 3.4 & $0.395-$ \\
\hline & 3 & 164.3 & $0.079-$ & 7.4 & $0.023 * *$ \\
\hline & 4 & ND & & ND & \\
\hline & 5 & ND & & ND & \\
\hline \multirow{5}{*}{ Adenosine } & 1 & 1.4 & $0.568-$ & 1.1 & $0.755-$ \\
\hline & 2 & 2.7 & $0.237-$ & 1.3 & $0.442-$ \\
\hline & 3 & 1.8 & $0.006 * * *$ & 1.5 & 0.055 - \\
\hline & 4 & 1.4 & $0.093-$ & 0.7 & 0.164 - \\
\hline & 5 & 1.2 & $0.098-$ & 0.8 & $0.273-$ \\
\hline \multirow[t]{5}{*}{ Pant } & 1 & 0.3 & $0.004 * * *$ & 0.8 & 0.607 - \\
\hline & 2 & 0.2 & $0.002 * * *$ & 0.2 & $0.0001 * * *$ \\
\hline & 3 & 0.4 & $0.038^{* *}$ & 0.2 & $0.0004 * * *$ \\
\hline & 4 & ND & & ND & \\
\hline & 5 & ND & & ND & \\
\hline
\end{tabular}

Untargeted metabolomics was performed on WT and $\Delta p u r H$ mutant of $S$. enterica grown in glucose minimal medium supplemented with adenine and thiamine. Relative concentrations of 3 key metabolites whose physiological changes have been previously described were extracted.

${ }^{a}$ Experimental replicates consisted of 3 biological replicates of each strain.

${ }^{b}$ Fold changes were the ratio of the average MS ion intensities (peak areas) in WT and $\Delta p u r H$ strains $(\Delta p u r H / W T)$.

${ }^{c} p$-values indicative of statistically significant differences are noted as follows $<0.05(* *),<0.01(* * *)$.

\section{Metabolomics patterns suggest a metabolic explanation for suppressor mutations}

Data from extensive in vivo experimentation determined that the accumulation of AICAR is responsible for the inability of a purH mutant to synthesize thiamine. We previously reported the isolation of loss-of-function mutations that restored thiamine synthesis in a purH mutant. The relevant loci ( $g d h A, m t f A, z w f$ and fumAC) are involved in central metabolic processes, and an explanation for their ability to restore thiamine synthesis was not immediately apparent [19] (Figure 5). A metabolomics approach was used to query the relevant metabolites in the suppressor strains compared to the parental purH mutant. When all experiments were considered, there was a trend indicating that some suppressor strains had less AICAR/AICARs than the parental purH mutant. Unfortunately, AICARs was not detected in 1 of the 2 experiments, and the conclusion about decreased pools was typically supported with statistically significant data in only one of the two experiments for each mutant. With these caveats, the metabolomics data indicated that lesions in $m t f A, z w f$ and fumAC appeared to decrease AICAR/AICARs concentrations to approximately half of that in the purH parent strain (Table 3). Thus, while they failed to provide any mechanistic information, these data were consistent with the fact that in- creased AICAR prevented thiamine synthesis in a purH mutant.

Significantly, pantothenate was increased in the zwf and fumAC mutants in late stationary phase samples, which suggested that pantothenate synthesis was partially restored by the decrease in AICAR caused by zwf and fu$m A C$ mutations. These data were consistent with lowered pantothenate being a downstream consequence of AICAR accumulation. Despite the generally appropriate trend, these data did not answer the question of whether, and how, such an apparently modest increase in pantothenate synthesis could increase the efficiency of the Thiccatalyzed step in thiamine synthesis. In contrast, the mtfA mutation decreased the AICAR/AICARs levels in the purH mutant, while also generating a further 10 -fold decrease (compared to $\mathrm{purH}$ mutant) in pantothenate levels in early stationary phase ( $p$-values $0.075,0.001$ ) and unchanged pantothenate levels in late stationary phase. These results were unexpected since they were not obviously consistent with the zwf and fumAC suppressor mutations that lower AICAR/AICARs. Whether this finding is due to a technical artifact, or predicts that MtfA modulates pantothenate levels will have to be explored experimentally. In contrast to all other suppressor mutations, AICAR, AICARs and pantothenate were not statistically changed by a gdhA muta- 

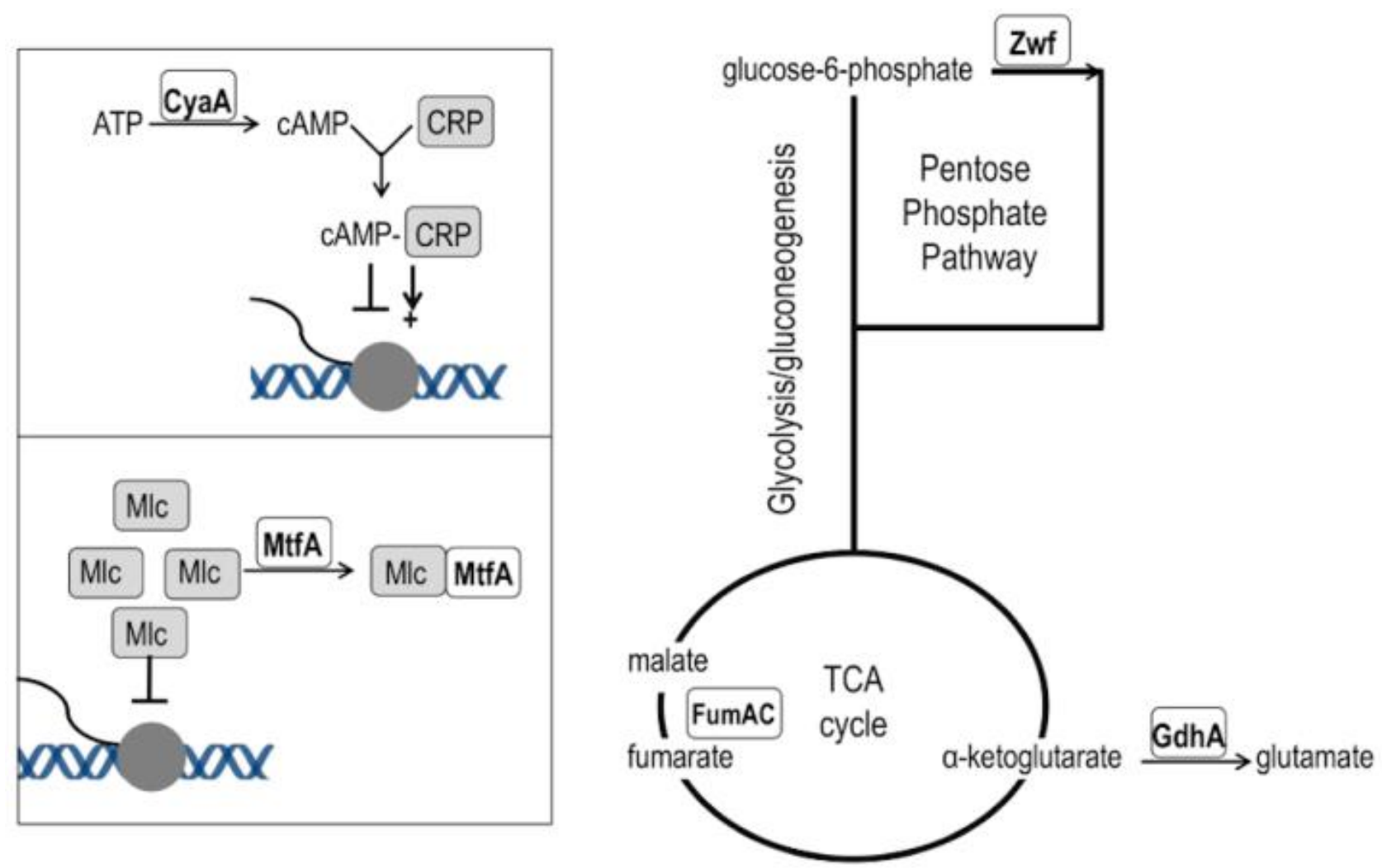

FIGURE 5: Network position of purH suppressor mutations. Loss-of-function mutations that suppress the thiamine requirement of a purH mutant have a role in various aspects of central carbon metabolism. The cyaA gene encodes adenylate cyclase and its product (cAMP) is the effector of the CRP transcriptional regulator that modulates $>100$ genes [45]. The $m t f A$ gene encodes the Mlc titration factor; Mlc is a global regulator of sugar metabolism. Both CAMP-CRP and MtfA are part of the PTS signal transduction pathway [46]. Fumarate dehydratase (fu$m A C)$ and glutamate dehydrogenase $(g d h A)$ function in, or just peripheral to, the TCA cycle. Glucose-6-phosphate dehydrogenase (zwf) is the first enzyme of the oxidative pentose phosphate pathway.

tion in any of the experiments and statistically significant changes in the respective metabolome were not obviously integrated with thiamine synthesis. Collectively these data suggest that $z w f$, fumAC and $m t f A$ suppressor mutations decrease AICAR/AICARs and restore thiamine synthesis via an effect downstream of pantothenate synthesis or by a mechanism unrelated to CoA. These data also suggest that the gdhA mutant employs a mechanism that circumvents elevated levels of AICAR present in a purH mutant. The complexity of the metabolomics data, while looking at only 3 metabolites, emphasizes the difficulty in deciphering the network connections without a phenotypic context. If the qualitative trends observed are significant, they suggest multiple paths to suppression exist. With confidence in the techniques used to generate them, the data facilitate the development of hypotheses that can be tested experimentally to extend our understanding of the metabolic system.

CAMP accumulates in a purH mutant and inhibits thiamine synthesis

Thiamine synthesis was restored in a purH mutant by a lesion in cyaA (encoding adenylate cyclase) [19]. When exogenous CAMP, the product of adenylate cyclase, was added to the medium the purH cyaA mutant strain again required thiamine (data not shown). These data suggested a model in which i) cAMP levels were elevated in a purH mutant and ii) the increased cAMP contributed to the inability of a purH mutant to generate sufficient thiamine for growth. Thus it was gratifying that the metabolomics data showed a 2.2-fold (avg. of statistically significant values) increase in cAMP levels in a purH mutant over those in wild-type (Table 4). Elevated cAMP in a purH mutant versus wild-type strain was independently verified using an ELISA assay, which detected a 2.1-fold difference ( $p$-value 0.004). These data supported a model where AICAR accumulation leads to elevated cAMP levels, which in turn modulates cellular metabolism, including the inhibition of thiamine biosynthesis.

\section{Identification of downstream effects of elevated cAMP}

An insertion deletion of the icc locus was made in an otherwise wild-type $S$. enterica strain to isolate the global effects of increased cAMP from those caused by increased AICAR levels. The icc mutant strain lacks cAMP phosphodiesterase activity and thus cannot catalytically degrade CAMP. In replicate experiments, elevated CAMP levels were observed in the untargeted metabolomics dataset of an icc mutant in early (3.0-fold) and late (1.9-fold) stationary phases. Importantly, the AICAR/AICARs pools in the icc mutant were unchanged from wild-type, which allowed 
TABLE 3. Changes in central carbon metabolism reverse consequences of purH deletion.

\begin{tabular}{|c|c|c|c|c|c|c|c|c|c|c|c|c|}
\hline \multirow[t]{2}{*}{ Genotype } & \multicolumn{4}{|c|}{ AICAR } & \multicolumn{4}{|c|}{ AICARs } & \multicolumn{4}{|c|}{ Pant } \\
\hline & ES1 & ES2 & LS1 & LS2 & ES1 & ES2 & LS1 & LS2 & ES1 & ES2 & LS1 & LS2 \\
\hline$\Delta p u r H^{a}$ & $367.2^{* * *}$ & $206.0 * *$ & $34.1^{* *}$ & $32.7 * * *$ & ND & 169.1 & ND & $7.6^{* *}$ & $0.2^{* * *}$ & $0.4^{* *}$ & $0.2^{* * *}$ & $0.2^{* * *}$ \\
\hline$\Delta p u r H$ gdh $A^{b}$ & 0.8 & 1.8 & 1.4 & 0.8 & ND & 1.4 & ND & 0.7 & 0.7 & 0.3 & 1.0 & 1.3 \\
\hline$\Delta$ purH mtfA $A^{b}$ & 0.7 & 0.6 & 0.6 & $0.4^{* *}$ & ND & 0.8 & ND & $0.1^{* *}$ & 0.1 & $0.1 * * *$ & 0.8 & 1.0 \\
\hline$\Delta p u r H z w f^{b}$ & 0.5 & 0.6 & 1.1 & 0.7 & ND & 0.1 & ND & $0.3^{* *}$ & 0.8 & 1.7 & 1.4 & $4.7^{* *}$ \\
\hline$\Delta p u r H$ fumA $C^{b}$ & $0.4^{* * *}$ & 0.6 & 0.5 & $0.5^{* *}$ & ND & 0.5 & ND & $0.2^{* *}$ & 1.1 & 1.1 & $3.2 * * *$ & $5.7 * * *$ \\
\hline
\end{tabular}

Metabolite fold changes were quantified in untargeted metabolomics analyses of WT and mutant strains of S. enterica. Cells were grown in glucose minimal medium supplemented with adenine and thiamine and harvested during early (ES) and late stationary (LS) phase growth phases in duplicate experiments, each consisting of 3 biological replicates.

${ }^{a}$ Fold changes were the ratio of the average MS ion intensities in WT and $\Delta p u r H$ strains $(\Delta p u r H /$ WT $)$.

${ }^{\mathrm{b}}$ Fold changes were the ratio of the average MS ion intensities in $\Delta$ purH and $\Delta$ purH double mutants $(\Delta p u r H$ mutX/ $\Delta p u r H)$.

${ }^{c} p$-values indicative of statistically significant differences are denoted by: ${ }^{* *}(<0.05),{ }^{* * *}(<0.01)$.

us to decouple global changes arising from elevated CAMP versus AICAR. Changes in the metabolome that resulted from the 2- to 3-fold increase in cAMP in the icc mutant were compared to changes present in a purH mutant. Several metabolites had similar trends in the purH and icc mutants, but it was particularly notable that their pantothenate pools were comparably reduced compared to wildtype (Table 5). AICAR and AICARs are direct inhibitors of the pantothenate biosynthetic enzyme PanC, which is presumed to be responsible for the decreased pantothenate (CoA) in the purH mutant. These data indicated the mechanism of decreased pantothenate differed in the two strains and suggested that pantothenate synthesis is compromised on multiple fronts (direct inhibition, regulatory modulation) when AICAR accumulates.

In addition to decreased pantothenate, the icc mutant displayed other metabolite trends of a $\mathrm{purH}$ mutant including decreases in amino acid precursors (isopropylmalate, leucine biosynthesis; phosphoserine, serine biosynthesis) and increases in deoxypyrimidines (dCMP, dUMP). In total, these data suggested that in a purH mutant elevated CAMP was responsible for, or contributed to, these changes. Further analysis showed that the two mutants only had a minority of their statistically significant changes in common. We reasoned that in the icc mutant, elevated CAMP perturbed the wild-type metabolome whereas in the purH mutant, we observed the effects of an elevated cAMP pool in the context of a very disrupted metabolism. Our results suggested that elevated CAMP, at most, accounted for $25 \%$ of statistically significant changes observed in a $\mathrm{purH} \mathrm{mu-}$ tant. This may in part reflect an inability of these approaches to adequately capture statistical significance. The icc mutant analysis allowed us to identify global effects of elevated CAMP pools independent of AICAR accumulation and thus defined the downstream effects of one of the major metabolic perturbations in a purH mutant. These results supported the potential of metabolomics analyses to modularize the pleotropic effects of genetic mutations.

TABLE 4. cAMP accumulates in a $\triangle p$ purH mutant.

\begin{tabular}{|c|c|c|c|c|c|c|c|}
\hline & \multicolumn{4}{|c|}{ Untargeted metabolomics $^{\mathrm{a}}$} & \multicolumn{3}{|c|}{ ELISA (pmol/mL) } \\
\hline Genotype & ES1 & ES2 & LS1 & LS2 & Rep & WT & $\Delta$ purH \\
\hline & \multicolumn{4}{|c|}{ Fold change (mutX/WT) } & & & \\
\hline$\Delta p u r H$ & 2.2 & $2.2^{* *}$ & 1.3 & $1.6^{* * *}$ & 1 & 28.5 & 57.0 \\
\hline \multirow[t]{2}{*}{$\Delta i c c$} & 2.7 & $3.3^{* * *}$ & 1.5 & 2.2 & 2 & 23.7 & 48.7 \\
\hline & \multicolumn{4}{|c|}{ Fold change ( $\Delta p u r H$ mutX/ $\Delta p u r H)$} & 3 & 25.6 & 58.6 \\
\hline$\Delta p u r H$ gdhA & 0.9 & 0.5 & 1.1 & 0.7 & Avg. & 25.9 & 54.7 \\
\hline$\Delta p u r H$ mtfA & 0.1 & $0.3^{* * *}$ & 1.0 & $0.7 * * *$ & & & \\
\hline$\Delta p u r H z w f$ & 0.4 & $0.3^{* * *}$ & $1.4^{* *}$ & $0.4 * * *$ & & Fold Change & 2.1 \\
\hline$\triangle$ purH fumAC & 0.8 & $0.6^{* *}$ & $0.5 * * *$ & 0.8 & & $p$-value & $0.004 * * *$ \\
\hline
\end{tabular}

cAMP levels were measured in WT and mutant strains of S. enterica by untargeted metabolomics (relative levels) or by a competitive enzyme-linked immunosorbent assay (ELISA). Cells were grown in minimal medium containing glucose, adenine and thiamine medium and harvested during early (ES) and/or late stationary (LS) phase (only harvested during ES for ELISA). Each experiment consisting of 3 biological replicates of each strain. Fold changes were the ratio of the average MS ion intensities between two strains.

${ }^{a} \mathrm{p}$-values indicative of statistically significant differences are denoted by: ${ }^{* *}(<0.05),{ }^{* * *}(<0.01)$. 


\section{Conclusions}

This study used the well-characterized purH mutant in $S$. enterica as a test case for metabolomics approaches to investigate bacterial physiology and to probe the global changes caused by accumulation of AICAR. AICAR is a purine biosynthetic intermediate that impacts a variety of cellular processes in diverse organisms [10, 20-28], and is the cause of the thiamine auxotrophy in a purH mutant of $S$. enterica. As a proof-of-principle, metabolomics data were evaluated for a handful of metabolites that are different in purH and wild-type strains $[10,14,15,20]$. With a few caveats an untargeted metabolomics approach captured known metabolic changes of a purH mutant, thus validating the general use of this approach.

The extent of the metabolic changes caused by removal of a single gene was unanticipated, and emphasized how dramatically AICAR levels changed the intracellular environment. Technical limitations prevented a statistically significant cataloging of all changes. To circumvent this, repeatable qualitative trends were evaluated in the context of biological knowledge and past experimental data. Despite the caveats with quantitative analyses, biological insights were gained from these metabolomics data. Firstly, an elevation of CAMP, predicted by previous genetic analyses, was detected in a purH mutant. The partial overlap of metabolic changes caused by a purH mutation and those detected in an icc mutant which lacks the cAMP phosphodiesterase, supported the modular description of the downstream effects that were due to CAMP accumulation rather than as a direct result of AICAR accumulation.

Secondly, mutations that suppressed the thiamine auxotrophy of a purH mutant caused metabolic changes that were not predictable from the framework of metabolism, but were consistent with moving the network in a $\mathrm{purH}$ mutant back toward a stable wild-type pattern. Specifically, mutations in $m t f A, z w f$, and fumAC decreased the levels of AICAR and CAMP compared to the parental purH mutant.

Previous studies have shown that AICAR affects diverse metabolic processes [10, 20, 23]. Metabolomics data obtained in this study confirmed that AICAR's physiological roles extend well beyond the PHT network in S. enterica. The data raised a number of issues that must be explored as we integrate biochemical genetic analysis with metabolomics approaches to understand the metabolic network in bacteria. For instance, what constitutes a physiologically relevant change in metabolite pool size? Is the 2 -fold decrease in AICAR found in the suppressor mutants significant enough to account for the phenotypic switch from auxotroph to prototroph? Finally, how does one sort through the extensive data that is obtained in a global study to identify relevant information in the absence of biological context for the analyses? The latter question is particularly challenging when the much of the data are not well-replicated even with rigorous experimentation. The current study benefited from our prior knowledge of the physiological system, which allowed us to implement $p$ values and trend metrics such that anticipated changes were detected. To our knowledge this study is the first of its kind to probe the physiological accuracy and reproducibility of metabolomics approaches to address specific metabolic questions in bacteria. The results were mixed, in that qualitative trends were detected, but statistically significant changes were less consistent. As is the case with many high-throughput studies and well put by Dalman et al. with

TABLE 5. Increased CAMP levels account for a subset of perturbations observed in a $\Delta$ purH mutant.

\begin{tabular}{|c|c|c|c|c|c|c|c|c|c|c|c|c|c|c|c|c|}
\hline & \multicolumn{8}{|c|}{ Early Stationary Phase } & \multicolumn{8}{|c|}{ Late Stationary Phase } \\
\hline & \multicolumn{4}{|c|}{ Exp 1} & \multicolumn{4}{|c|}{$\operatorname{Exp} 2$} & \multicolumn{4}{|c|}{ Exp 1} & \multicolumn{4}{|c|}{ Exp 2} \\
\hline & \multicolumn{2}{|c|}{$\Delta$ purH/WT } & \multicolumn{2}{|c|}{$\Delta i c c / W T$} & \multicolumn{2}{|c|}{$\Delta p u r H / W T$} & \multicolumn{2}{|c|}{$\Delta i c c / W T$} & \multicolumn{2}{|c|}{$\Delta p u r H / W T$} & \multicolumn{2}{|c|}{$\Delta i c c / W T$} & \multicolumn{2}{|c|}{$\Delta$ purH/WT } & \multicolumn{2}{|c|}{$\Delta i c c / W T$} \\
\hline & FC & $\mathrm{p}$-val & FC & $\mathrm{p}$-val & FC & p-val & FC & p-val & FC & p-val & FC & $\mathrm{p}$-val & FC & p-val & FC & p-val \\
\hline CAMP & 2.2 & 0.165 & 2.7 & 0.293 & 2.2 & 0.035 & 3.3 & 0.004 & 1.3 & 0.105 & 1.5 & 0.184 & 1.6 & 0.005 & 2.2 & 0.098 \\
\hline Pantothenate & 0.2 & 0.002 & 0.6 & 0.367 & 0.4 & 0.038 & 0.2 & 0.008 & 0.2 & 0.000 & 0.2 & 0.000 & 0.2 & 0.000 & 0.6 & 0.128 \\
\hline Isopropylmalate & 0.1 & 0.005 & 0.4 & 0.080 & 0.1 & 0.073 & 0.2 & 0.098 & 4.1 & 0.002 & 0.4 & 0.008 & 0.6 & 0.068 & 0.4 & 0.063 \\
\hline Phosphoserine & 0.0 & 0.030 & 0.4 & 0.079 & 0.1 & 0.023 & 0.4 & 0.033 & 5.1 & 0.048 & 0.7 & 0.376 & 1.1 & 0.756 & 0.7 & 0.568 \\
\hline Adenine & 0.7 & 0.149 & 0.4 & 0.014 & 0.8 & 0.287 & 0.7 & 0.338 & 1.5 & 0.038 & 0.2 & 0.011 & 1.2 & 0.588 & 3.2 & 0.339 \\
\hline CMP & 0.4 & 0.061 & 0.3 & 0.033 & 0.6 & 0.015 & 1.6 & 0.284 & 0.5 & 0.016 & 0.5 & 0.028 & 0.6 & 0.063 & 0.3 & 0.008 \\
\hline $\mathrm{dCMP}$ & 6.1 & 0.025 & 1.9 & 0.336 & 3.1 & 0.021 & 0.9 & 0.834 & 1.3 & 0.036 & 1.7 & 0.347 & 1.2 & 0.693 & 0.3 & 0.100 \\
\hline dUMP & 22.2 & 0.048 & 2.7 & 0.180 & 8.9 & 0.014 & 1.0 & 0.968 & 1.1 & 0.843 & 1.5 & 0.208 & 1.1 & 0.721 & 1.6 & 0.181 \\
\hline DHAP & 1.7 & 0.144 & 0.5 & 0.147 & 4.5 & 0.017 & 1.7 & 0.105 & 2.3 & 0.031 & 4.4 & 0.208 & 1.7 & 0.129 & 1.9 & 0.332 \\
\hline UDP-Glucose & 13.2 & 0.086 & 3.9 & 0.239 & 2.0 & 0.189 & 3.0 & 0.107 & 3.0 & 0.050 & 14.0 & 0.002 & 0.5 & 0.428 & 5.0 & 0.198 \\
\hline Ribose 5-P & 5.0 & 0.124 & 1.1 & 0.780 & 1.7 & 0.033 & 1.4 & 0.198 & 2.1 & 0.078 & 3.8 & 0.142 & 1.5 & 0.103 & 1.4 & 0.417 \\
\hline Hydroxyisocaproate & 1.9 & 0.314 & 0.9 & 0.743 & 20.2 & 0.020 & 1.3 & 0.155 & 1.3 & 0.089 & 1.7 & 0.263 & 6.2 & 0.002 & 7.6 & 0.299 \\
\hline Carbamoyl-P & 3.5 & 0.155 & 1.3 & 0.628 & 6.5 & 0.042 & 2.0 & 0.307 & 0.5 & 0.330 & 2.3 & 0.086 & 0.4 & 0.091 & 1.6 & 0.097 \\
\hline Glu & 1.2 & 0.811 & 1.1 & 0.909 & 2.4 & 0.003 & 4.8 & 0.051 & 1.2 & 0.785 & 1.8 & 0.040 & 0.9 & 0.518 & 0.9 & 0.647 \\
\hline Met & 0.5 & 0.001 & 0.2 & 0.015 & 0.2 & 0.053 & 0.3 & 0.063 & 1.3 & 0.609 & 0.6 & 0.408 & 0.6 & 0.052 & 1.0 & 0.837 \\
\hline Aminoadipate & 2.5 & 0.114 & 1.1 & 0.837 & 2.7 & 0.001 & 1.4 & 0.164 & 1.8 & 0.005 & 2.2 & 0.012 & 2.0 & 0.021 & 1.0 & 0.753 \\
\hline Phenylpyruvate & 3.1 & 0.155 & 4.2 & 0.038 & 2.3 & 0.079 & 2.9 & 0.309 & 1.2 & 0.594 & 1.4 & 0.186 & 1.2 & 0.823 & 1.6 & 0.451 \\
\hline Phe & 6.9 & 0.083 & 3.0 & 0.226 & 2.9 & 0.020 & 8.7 & 0.314 & 0.7 & 0.018 & 1.7 & 0.069 & 0.8 & 0.276 & 0.7 & 0.200 \\
\hline Tyr & 4.1 & 0.128 & 4.4 & 0.306 & 1.8 & 0.069 & 6.0 & 0.317 & 0.7 & 0.081 & 1.4 & 0.040 & 0.8 & 0.154 & 1.0 & 0.927 \\
\hline Adenosine & 2.7 & 0.237 & 1.5 & 0.118 & 1.8 & 0.006 & 1.5 & 0.070 & 1.3 & 0.442 & 1.9 & 0.055 & 1.5 & 0.055 & 1.0 & 0.868 \\
\hline XMP & 1.3 & 0.690 & 2.4 & 0.335 & 1.9 & 0.018 & 0.4 & 0.047 & ND & & 12.9 & 0.372 & 0.2 & 0.461 & 0.2 & 0.424 \\
\hline Dihydroorotate & 5.1 & 0.010 & 1.9 & 0.210 & 3.1 & 0.179 & 2.1 & 0.307 & 1.7 & 0.312 & 5.6 & 0.242 & 1.2 & 0.176 & 3.5 & 0.297 \\
\hline Uridine & ND & & ND & & 37.4 & 0.001 & 4.1 & 0.452 & ND & & ND & & 5.0 & 0.010 & 2.5 & 0.576 \\
\hline CDP & 0.3 & 0.012 & 0.3 & 0.006 & 0.5 & 0.088 & 0.8 & 0.497 & 0.5 & 0.208 & 1.1 & 0.679 & 0.5 & 0.113 & 0.6 & 0.136 \\
\hline Adenylyl sulfate & 3.2 & 0.242 & 9.5 & 0.033 & 1.9 & 0.141 & 3.1 & 0.028 & 1.3 & 0.472 & 1.2 & 0.684 & 1.8 & 0.066 & 2.3 & 0.256 \\
\hline
\end{tabular}


regard to transcriptomic analyses, data interpretation is "more of an art than a science, with follow up gene expression studies a must" [44]. In the absence of this biological context, conclusions from qualitative metabolomics data must be made cautiously and with a clear understanding of what types of information can be gleaned from the data. Although untargeted and targeted metabolomics techniques are powerful tools, metabolite concentrations alone are not sufficient to fully describe cellular physiology; and the power of metabolomics is often better realized in combination with well-defined genetic systems and/or other data-enabled omics techniques.

\section{MATERIALS AND METHODS}

\section{Bacterial strains, media, and chemicals}

Strains used in this study are derivatives of $S$. enterica serovar Typhimurium LT2 and are described in Table S1. Rich medium consisted of Difco nutrient broth (NB) (8g/L) and $\mathrm{NaCl}(5 \mathrm{~g} / \mathrm{L})$. Defined medium consisted of No-carbon $\mathrm{E}$

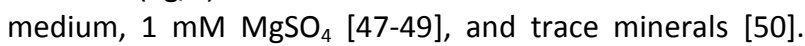
Glucose (11 mM) was used as the sole carbon source. Difco BiTek agar ( $15 \mathrm{~g} / \mathrm{L})$ was added for solid medium. Nutrients were supplemented at the following concentrations: adenine $(0.4 \mathrm{mM})$ thiamine $(0.1 \mu \mathrm{M})$. Chemicals were purchased from Sigma-Aldrich.

\section{Growth quantitation}

The cells from overnight cultures (NB or defined medium) were pelleted and resuspended in an equal volume of $0.85 \% \mathrm{NaCl}$ or for metabolomics samples, directly subcultured into the fresh defined medium. Cell density was measured as absorbance at $650 \mathrm{~nm}$ in a Spectronic 20 and reported as final yield at a given time.

\section{Genetic techniques}

Transductional crosses were performed with a highfrequency general transducing mutant of bacteriophage P22 (HT105/1, int-201) [51]. Methods for transductional crosses, isolation of transductants from bacteriophage, and identification of bacteriophage-free transductants have been previously described [52]. Mutant strains were constructed using standard genetic techniques. Insertion deletions were generated using the $\lambda$-red-mediated homologous recombination method and verified with PCR and sequence analysis [53].

\section{Cyclic AMP quantification}

Intracellular cyclic AMP was measured using enzyme-linked immunosorbent assay (ELISA) purchased from Cayman Chemical. Cyclic AMP concentrations were calculated using the manufacturer's provided data analysis tools.

\section{Metabolomics analysis}

Three $1 \mathrm{~mL}$ cultures (minimal glucose adenine thiamine medium) of each strain were grown at $37^{\circ} \mathrm{C}$ with shaking overnight ( $12 \mathrm{hr}$ ). In duplicate or triplicate, a $200 \mu \mathrm{L}$ aliquot of each overnight culture was used to inoculate $6 \mathrm{~mL}$ of fresh media and cultures were returned to identical growth conditions. Cell density was measured as absorb- ance at $650 \mathrm{~nm}\left(A_{650}\right)$. Once cells reached desired growth phase one culture of each biological replicate was used for metabolite extraction. Cell densities for mid-exponential phase and early stationary phases were, $A_{650}=0.45-0.55$ and $A_{650}=1.5-1.8$, respectively. Early stationary phase was defined as $1 \mathrm{~h}$ after maximum $A_{650}$ (1.5-1.8) was achieved. Late stationary phase was defined as $7 \mathrm{~h}$ after maximum $A_{650}$ was achieved. In each experiment, all strains and biological replicates were grown and processed simultaneously.

\section{Metabolite extraction.}

Metabolite extraction was performed generally as described [35]. Cells were harvested by vacuum filtering $5 \mathrm{~mL}$ aliquots of each culture through Magna, nylon supported, plain 0.45 micron, $47 \mathrm{~mm}$ filter. Filters were transferred (cell side down) into petri dishes containing $1.3 \mathrm{~mL}$ of extraction solvent (40:40:20 HPLC grade methanol, acetonitrile, water with $0.1 \%$ formic acid) chilled to $-20^{\circ} \mathrm{C}$. The extraction was allowed to proceed for $15 \mathrm{~min}$ at $-20^{\circ} \mathrm{C}$. At $4^{\circ} \mathrm{C}$, and cell side of the filters were rinsed by repeatedly pipetting the extraction solvent over the face of the filters. Extracts were transferred to $1.5 \mathrm{~mL}$ centrifuge tubes. Filters were washed a second time with $300 \mu \mathrm{L}$ of new extraction fluid. Filters were then discarded and extracts were added to initial extracts in $1.5 \mathrm{~mL}$ centrifuge tubes. Extracts were centrifuged for 5 minutes (16.1 rcf) at $4^{\circ} \mathrm{C}$ and the supernatant was transferred to glass vials. The pelleted cell debris was resuspended in $50 \mu \mathrm{L}$ of extraction solvent. The extraction was allowed to proceed for $15 \mathrm{~min}$ at $-20^{\circ} \mathrm{C}$. Samples were centrifuged for $5 \mathrm{~min}$ (16.1 rcf) at $4^{\circ} \mathrm{C}$. The supernatant was added to the appropriate glass vials to combine extracts. A final extraction of the pelleted cell debris was repeated as in the previous extraction. Vials containing all of the collected supernatant were dried to completion under a stream of $\mathrm{N}_{2}$ at room temperature. Solid residue was resuspended in $300 \mu \mathrm{L}$ of sterile water and transferred to $300 \mu \mathrm{L}$ autosampler vials.

\section{UHPLC-HRMS.}

Samples were kept at $4^{\circ} \mathrm{C}$ in an Ultimate $3000 \mathrm{RS}$ autosampler (Dionex, Sunnyvale, CA) until time of run. A 10 $\mu \mathrm{L}$ aliquot was injected and separated on a Synergi $2.5 \mu$ Hydro-RP 100, $100 \times 2.00 \mathrm{~mm}$. Untargeted metabolomics analysis was performed via a method adapted from Lu et al. 2010 [36]. The chromatographic gradient was from 0 to 5 $\min 0 \% \mathrm{~B}$, from 5 to $13 \mathrm{~min} 20 \% \mathrm{~B}$, from 13 to $15.5 \mathrm{~min}$ $55 \% \mathrm{~B}$, from 15.5 to $19 \mathrm{~min} 95 \% \mathrm{~B}$, and from 19 to $25 \mathrm{~min}$ $0 \%$ B. Solvent A was 97:3 HPLC grade water:methanol, 10 $\mathrm{mM}$ tributylamine, and $15 \mathrm{mM}$ acetic acid. Solvent $B$ was HPLC grade methanol. The flow rate and column compartment temperature were held constant at $200 \mu \mathrm{L} / \mathrm{min}$ and $25^{\circ} \mathrm{C}$, respectively. Following chromatographic elution, the samples were ionized via electrospray ionization in negative ion mode using a spray voltage of $3 \mathrm{kV}$, nitrogen sheath gas of 10 (arbitrary units), capillary temperature of $320^{\circ} \mathrm{C}$, and AGC target set to $3 \mathrm{e} 6$. Data was acquired using an Exactive Plus Orbitrap mass spectrometer (Thermo Scientific, Waltham, MA). The orbitrap was run in full scan 
mode from 85 to $800 \mathrm{~m} / \mathrm{z}$ from 0 to $9 \mathrm{~min}$ and from 100 to $1000 \mathrm{~m} / z$ from 9 to $25 \mathrm{~min}$ using a resolution of 140,000 .

\section{Data analysis.}

Raw MS data files were converted to $\mathrm{mzML}$ files using ProteoWizard software $[54,55]$. Converted spectra were processed using Metabolomic Analysis and Visualization Engine (MAVEN) [37]. Metabolites were identified based on exact mass ( $\pm 5 \mathrm{ppm}$ ) and retention times from methods included with MAVEN [36] as well as additional in-house validated metabolites. Additional features were putatively assigned based on $m / z$ and pathway analysis. For each of the "known" metabolites, extracted ion chromatograms were visualized by MAVEN and or manually integrated; Acceptable peak criteria were Guassian shaped with at least $\mathrm{S} / \mathrm{N}$ and signal to blank $>3$. In a given experiment, peak areas from biological triplicates were averaged and the ratios of the averages were reported as fold changes between two strains. To determine statistical significance of fold changes, an unpaired Student's t-test (two-tailed) was applied to peak area values of biological replicates. A p-value threshold of 0.05 was used. In instances where a metabolite was only detected in 2 of 3 samples, the zero value was excluded from all calculations.

Trends were defined as instances where one or more experimental replicate illustrated statistical significance and as a whole, the majority of experiments reflected fold changes in the same direction $(<0.8$ or $>1.2)$. In some instances trends are clear in one growth phase but absent or distinct in another; as these data are derived from distinct physiological scenarios they are not in conflict and both datasets may be presented for a more complete picture of the metabolome.

Partial Least Square Discriminate Analysis (PLSDA) was used as a multivariate analysis tool to provide insight into

\section{REFERENCES}

1. Koenigsknecht MJ, Downs DM (2010). Thiamine biosynthesis can be used to dissect metabolic integration. Trends Microbiol 18(6): 240247. doi: 10.1016/j.tim.2010.03.003

\section{Downs DM (2006). Understanding microbial metabolism. Annu Rev Microbiol 60:533-559.

10.1146/annurev.micro.60.080805.142308.

3. Koenigsknecht MJ, Lambrecht JA, Fenlon LA, Downs DM (2012). Perturbations in Histidine Biosynthesis Uncover Robustness in the Metabolic Network of Salmonella enterica. PLoS One 7(10): e48207. doi: 10.1371/journal.pone.0048207

4. Browne BA, Ramos Al, Downs DM (2006). PurF-independent phosphoribosyl amine formation in yjgF mutants of Salmonella enterica utilizes the tryptophan biosynthetic enzyme complex anthranilate synthase-phosphoribosyltransferase. J Bacteriol 188(19): 6786-6792. PMID:16980480

5. Enos-Berlage JL, Langendorf MJ, Downs DM (1998). Complex metabolic phenotypes caused by a mutation in $y j g F$, encoding a member of the highly conserved YER057c/YjgF family of proteins. J Bacteriol 180(24): 6519-6528. PMID:9851994

6. Lambrecht JA, Browne BA, Downs DM (2010). Members of the YjgF/YER057c/UK114 family of proteins inhibit phosphoribosylamine the unique metabolic fingerprint of the purH mutant. The 179 metabolites that were identified in the untargeted approach, were used in a pairwise fashion to generate plots and variable importance in projections (VIP) scores for the three growth phases tested. Further information can be extracted by collecting all metabolites that have VIP score greater than $1[56,57]$. The DiscriMiner package in $R$ was used to perform all statistical functions, and gglplot2 was used to visualize the results [58].

\section{ACKNOWLEDGMENTS}

This work was supported by NSF competitive grants MCB1411672 and MCB1615373 to DMD and SRC.

\section{SUPPLEMENTAL MATERIAL}

All supplemental data for this article are available online at www.microbialcell.com.

\section{CONFLICT OF INTEREST}

The authors have no conflict of interest to declare.

\section{COPYRIGHT}

(C) 2017 Bazurto et al. This is an open-access article released under the terms of the Creative Commons Attribution (CC BY) license, which allows the unrestricted use, distribution, and reproduction in any medium, provided the original author and source are acknowledged.

Please cite this article as: Jannell V. Bazurto, Stephen P. Dearth, Eric D. Tague, Shawn R. Campagna and Diana M. Downs (2017). Untargeted metabolomics confirms and extends the understanding of the impact of aminoimidazole carboxamide ribotide (AICAR) in the metabolic network of Salmonella enterica. Microbial Cell 5(2): 74-87. doi: 10.15698/mic2018.02.613

synthesis in vitro. J Biol Chem 285(45): 34401-34407. doi 10.1074/jbc.M110.160515

7. Schmitz G, Downs DM (2004). Reduced Transaminase B (IlvE) Activity Caused by the Lack of yjgF Is Dependent on the Status of Threonine Deaminase (IlvA) in Salmonella enterica Serovar Typhimurium. J Bacteriol 186(3): 803-810. doi: 10.1128/jb.186.3.803810.2004

8. Ernst DC, Downs DM (2015). The STM4195 gene product (PanS) transports coenzyme A precursors in Salmonella enterica. J Bacteriol 197(8): 1368-1377. doi: 10.1128/JB.02506-14

9. Frodyma M, Rubio A, Downs DM (2000). Reduced flux through the purine biosynthetic pathway results in an increased requirement for coenzyme $\mathrm{A}$ in thiamine synthesis in Salmonella enterica serovar Typhimurium. J Bacteriol 182(1): 236-240. PMID:10613889

10. Bazurto JV, Downs DM (2014). Amino-4-imidazolecarboxamide ribotide directly inhibits coenzyme A biosynthesis in Salmonella enterica. J Bacteriol 196(4): 772-779. doi: 10.1128/JB.01087-13

11. Dougherty MJ, Downs DM (2006). A connection between ironsulfur cluster metabolism and the biosynthesis of 4-amino-5hydroxymethyl-2-methylpyrimidine pyrophosphate in Salmonella enterica. Microbiol 152(Pt 8): 2345-2353. PMID:16849799 
12. Bazurto JV, Farley KR, Downs DM (2016). An Unexpected Route to an Essential Cofactor: Escherichia coli Relies on Threonine for Thiamine Biosynthesis. mBio 7(1): e01840-01815. doi: 10.1128/mBio.01840-15

13. Zhang K, Bian J, Deng Y, Smith A, Nunez RE, Li MB, Pal U, Yu AM, Qiu W, Ealick SE, Li C (2016). Lyme disease spirochaete Borrelia burgdorferi does not require thiamin. Nat Microbiol 2:16213. doi: 10.1038/nmicrobiol.2016.213

14. Yura $T$ (1956). Evidence of nonidentical alleles in purine requiring mutants of Salmonella typhimurium. Publications of the Carnegie Institution 612:63-75.

15. Newell PC, Tucker RG (1968). Biosynthesis of the pyrimidine moiety of thiamine. A new route of pyrimidine biosynthesis involving purine intermediates. Biochem J 106(1): 279-287. PMID:4889364

16. Allen S, Zilles JL, Downs DM (2002). Metabolic flux in both the purine mononucleotide and histidine biosynthetic pathways can influence synthesis of the hydroxymethyl pyrimidine moiety of thiamine in Salmonella enterica. J Bacteriol 184(22): 6130-6137. PMID:12399482

17. Palmer LD (2014). Characterization of Thiamine Biosynthetic Enzymes and Their Integration in the Metabolic Network of Salmonella enterica. PhD thesis, University of Georgia, Athens, GA.

18. Gralnick J, Webb E, Beck B, Downs D (2000). Lesions in gshA (encoding gamma-L-glutamyl-L-cysteine synthetase) prevent aerobic synthesis of thiamine in Salmonella enterica serovar typhimurium LT2. J Bacteriol 182(18): 5180-5187. PMID:10960103

19. Bazurto JV, Heitman NJ, Downs DM (2015). Aminoimidazole Carboxamide Ribotide Exerts Opposing Effects on Thiamine Synthesis in Salmonella enterica. J Bacteriol 197(17): 2821-2830. doi: 10.1128/JB.00282-15

20. Kuramitsu HK, Udaka S, Moyed HS (1964). Induction of Inosine 5'Phosphate Dehydrogenase and Xanthosine 5'-Phosphate Aminase by Ribosyl-4-Amino-5-Imidazolecarboxamide in Purine-Requiring Mutants of Escherichia coli B. J Biol Chem 239(3425-3430. PMID:14245398

21. Burton K (1971). Limitation of Glycolysis in Adenine-Deficient Escherichia coli. Biochem J 123(4): 585. PMID:4942450

22. Rebora K, Laloo B, Daignan-Fornier B (2005). Revisiting purinehistidine cross-pathway regulation in Saccharomyces cerevisiae: a central role for a small molecule. Genetics 170(1): 61-70. doi: 10.1534/genetics.104.039396

23. Dougherty MJ, Boyd JM, Downs DM (2006). Inhibition of fructose1,6-bisphosphatase by aminoimidazole carboxamide ribotide prevents growth of Salmonella enterica purH mutants on glycerol. J Biol Chem 281(45): 33892-33899. doi: 10.1074/jbc.M604429200

24. Sullivan JE, Brocklehurst KJ, Marley AE, Carey F, Carling D, Beri RK (1994). Inhibition of lipolysis and lipogenesis in isolated rat adipocytes with AICAR, a cell-permeable activator of AMP-activated protein kinase. FEBS Lett 353(1): 33-36. PMID:7926017

25. Pinson B, Vaur S, Sagot I, Coulpier F, Lemoine S, Daignan-Fornier B (2009). Metabolic intermediates selectively stimulate transcription factor interaction and modulate phosphate and purine pathways. Genes \& Development 23(12): 1399-1407. doi: 10.1101/gad.521809

26. Daignan-Fornier B, Pinson B (2012). 5-Aminoimidazole-4carboxamide-1-beta-D-ribofuranosyl 5'-Monophosphate (AICAR), a Highly Conserved Purine Intermediate with Multiple Effects. Metabolites 2(2): 292-302. doi: 10.3390/metabo2020292

27. Ha TM, Morgan SL, Vaughn WH, Baggott JE (1992). Inhibition of adenosine deaminase and S-adenosylhomocysteine hydrolase by 5 - aminoimidazole-4-carboxamide riboside (AICAR). FASEB 6(4): A1215A1215.

28. Kim PB, Nelson JW, Breaker RR (2015). An ancient riboswitch class in bacteria regulates purine biosynthesis and one-carbon metabolism. Mol Cell 57(2): 317-328. doi: 10.1016/j.molcel.2015.01.001

29. Bazurto JV, Downs DM (2011). Plasticity in the purine-thiamine metabolic network of Salmonella. Genetics 187(2): 623-631. doi: 10.1534/genetics.110.124362

30. Magasanik B, Karibian D (1960). Purine nucleotide cycles and their metabolic role. J Biol Chem 235:2672-2681. PMID:14419770

31. Moyed HS, Magasanik B (1960). The biosynthesis of the imidazole ring of histidine. J Biol Chem 235:149-153. PMID:14424819

32. Downs DM, Roth JR (1991). Synthesis of thiamine in Salmonella typhimurium independent of the purF function. J Bacteriol 173(20): 6597-6604. PMID:1917881

33. Johnston HM, Roth JR (1979). Histidine mutants requiring adenine: selection of mutants with reduced his expression in Salmonella typhimurium. Genetics 92:1-15. PMID:387516

34. Winkler ME (1987). Biosynthesis of histidine. In: Neidhardt FC, editor Escherichia coli and Salmonella typhimurium, cellular and molecular biology. ASM, Washingtion, D.C.; pp 395-411.

35. Rabinowitz JD, Kimball E (2007). Acidic acetonitrile for cellular metabolome extraction from Escherichia coli. Anal Chem 79(16): 6167-6173. doi: 10.1021/ac070470c

36. Lu W, Clasquin MF, Melamud E, Amador-Noguez D, Caudy AA, Rabinowitz JD (2010). Metabolomic analysis via reversed-phase ionpairing liquid chromatography coupled to a stand alone orbitrap mass spectrometer. Anal Chem 82(8): 3212-3221. doi: 10.1021/ac902837x

37. Clasquin MF, Melamud E, Rabinowitz JD (2012). LC-MS data processing with MAVEN: a metabolomic analysis and visualization engine. Curr Protoc Bioinformatics Chapter 14: Unit14 11. doi: 10.1002/0471250953.bi1411s37

38. Andrews SC, Robinson AK, Rodriguez-Quinones F (2003). Bacterial iron homeostasis. FEMS Microbiol Rev 27(2-3): 215-237. PMID:12829269

39. Booth IR (1985). Regulation of cytoplasmic $\mathrm{pH}$ in bacteria. Microbiol Rev 49(4): 359-378. PMID:3912654

40. Parsons JB, Rock CO (2013). Bacterial lipids: metabolism and membrane homeostasis. Prog Lipid Res 52(3): 249-276. doi: 10.1016/j.plipres.2013.02.002

41. Rohlman CE, Matthews RG (1990). Role of purine biosynthetic intermediates in response to folate stress in Escherichia coli. J Bacteriol 172(12): 7200-7210. PMID:2254281

42. Gots JS, Dalal FR, Shumas SR (1969). Genetic eparation of the inosinic acid cyclohydrolase-transformylase complex of Salmonella typhimurium. J Bacteriol 99(2): 441-449. PMID:4897111

43. Palmer LD, Downs DM (2013). The Thiamine Biosynthetic Enzyme ThiC Catalyzes Multiple Turnovers and Is Inhibited by SAdenosylmethionine (AdoMet) Metabolites. J Biol Chem 288(42): 30693-30699. doi: 10.1074/jbc.M113.500280

44. Dalman MR, Deeter A, Nimishakavi G, Duan ZH (2012). Fold change and p-value cutoffs significantly alter microarray interpretations. BMC bioinformatics 13 Suppl 2: S11. doi: 10.1186/1471-2105-13-S2-S11

45. Kolb A, Busby S, Buc H, Garges S, Adhya S (1993). Transcriptional regulation by CAMP and its receptor protein. Annual Rev Biochem 62:749-795. doi: 10.1146/annurev.bi.62.070193.003533 
46. Becker AK, Zeppenfeld T, Staab A, Seitz S, Boos W, Morita T, Aiba $\mathrm{H}$, Mahr K, Titgemeyer $\mathrm{F}$, Jahreis $\mathrm{K}$ (2006). Yeel, a novel protein involved in modulation of the activity of the glucosephosphotransferase system in Escherichia coli K-12. J Bacteriol 188(15): 5439-5449. doi: 10.1128/JB.00219-06

47. Vogel HJ, Bonner DM (1956). Acetylornithase of Escherichia coli: partial purification and some properties. J Biol Chem 218:97-106. PMID:13278318

48. Berkowitz D, Hushon JM, Whitfield HJ, Roth J, Ames BN (1968). Procedure for identifying nonsense mutations. J Bacteriol 96:215-220. PMID:4874308

49. Davis RW, Botstein D, Roth JR, Cold Spring Harbor Laboratory. (1980). Advanced bacterial genetics. Cold Spring Harbor Laboratory, Cold Spring Harbor, N.Y.

50. Balch W, E.; Wolfe, R. S. (1976). New approach to the cultivation of methanogenic bacteria: 2-mercaptoethanesulfonic acid (HS-CoM)dependent growth of Methanobacterium ruminantium in a pressurized atmosphere. Appl Environ Microbiol 32(6): 781-791. PMID:827241

51. Schmieger H (1972). Phage P22-mutants with increased or decreased transduction abilities. Mol Gen Genet 119(1): 75-88. PMID:4564719

52 Chan RK, Botstein D, Watanabe T, Ogata Y (1972). Specialized transduction of tetracycline resistance by phage P22 in Salmonella typhimurium. II Properties of a high transducing lysate. Virology 50:883-898. PMID:4565618

53. Datsenko KA, Wanner BL (2000). One-step inactivation of chromosomal genes in Escherichia coli K-12 using PCR products. Proc Natl Acad Sci USA 97(12): 6640-6645. PMID:10829079
54. Martens L, Chambers M, Sturm M, Kessner D, Levander $F$, Shofstahl J, Tang WH, Rompp A, Neumann S, Pizarro AD, MontecchiPalazzi L, Tasman N, Coleman M, Reisinger F, Souda P, Hermjakob H, Binz PA, Deutsch EW (2011). mzML--a community standard for mass spectrometry data. Molecular \& Cellular Proteomics : MCP 10(1): R110 000133. doi: 10.1074/mcp.R110.000133

55. Chambers MC, Maclean B, Burke R, Amodei D, Ruderman DL, Neumann S, Gatto L, Fischer B, Pratt B, Egertson J, Hoff K, Kessner D, Tasman N, Shulman N, Frewen B, Baker TA, Brusniak MY, Paulse C, Creasy D, Flashner L, Kani K, Moulding C, Seymour SL, Nuwaysir LM, Lefebvre B, Kuhlmann F, Roark J, Rainer P, Detlev S, Hemenway T, et al. (2012). A cross-platform toolkit for mass spectrometry and proteomics. Nat Biotechnol 30(10): 918-920. doi: 10.1038/nbt.2377

56. Li XZ, Yang XY, Wang Y, Zhang SN, Zou W, Wang Y, Li XN, Wang LS, Zhang ZG, Xie LZ (2017). Urine metabolic profiling for the pathogenesis research of erosive oral lichen planus. Arch Oral Biol 73:206-213. doi: 10.1016/j.archoralbio.2016.10.014

57. Xia J, Wishart DS (2011). Web-based inference of biological patterns, functions and pathways from metabolomic data using MetaboAnalyst. Nat Protoc 6(6): 743-760. doi: 10.1038/nprot.2011.319

58. Aad G, Abajyan T, Abbott B, Abdallah J, Khalek SA, Abdelalim AA, Abdinov O, Aben R, Abi B, Abolins M, AbouZeid OS, Abramowicz H, Abreu H, Acharya BS, Adamczyk L, Adams DL, Addy TN, Adelman J, Adomeit S, Adragna P, Adye T, Aefsky S, Aguilar-Saavedra JA, Agustoni M, Ahlen SP, Ahles F, Ahmad A, Ahsan M, Aielli G, Akesson TPA, et al. (2013). Search for a light charged Higgs boson in the decay channel $\mathrm{H}+$ -> c(s)over-bar in $\mathrm{t}(\mathrm{t})$ over-bar events using $\mathrm{pp}$ collisions at root $\mathrm{s}=7$ TeV with the ATLAS detector. Eur Phys J C 73(6). doi: 10.1140/epjc/s10052-013-2465-z 